\title{
Preface: Liver Cancer and Its Therapy
}

Liver cancer is a heterogenous malignant disease. This aggressive tumor is the third leading cause of cancer-related mortality, which is often higher in men than in women. The primary liver cancer, hepatocellular carcinoma (HCC), is mainly attributed to the prevalent hepatitis B viral infection and cirrhosis. Lack of specific symptoms and diagnostic biomarkers contribute to the failure to diagnose this malignancy in its early stages; it is often diagnosed at an advanced stage. Surgical techniques, such as radical resection and liver transplantation, can be effective; however, the shortage of organs for transplantation limits these applications. Regardless of the numerous advanced technologies available, the prognosis rate remains low and the death rate remains high in HCC. Thus, novel therapeutic strategies are urgently needed, including molecular targeted therapies as well as efficient biomarkers to diagnose the tumor in its early stages. In this issue, we have compiled the most current information regarding many of the essential novel therapies and medicinal strategies that are currently available to treat HCC.

Here, we focus on understanding HCC progression at the molecular level. The tumor microenvironment (TME) contributes to the progression of liver cancer and is a key player in liver cancer therapy. A clear understanding of the TME, its components, including cancer-associated fibroblasts, immune cells, tumor-associated macrophages, and stroma, as well as their specific roles, could represent possible therapeutic strategies. This information will contribute to the development of novel biomarkers that assist in the early diagnosis of tumor progression. Moreover, the articles in this issue also provide an in-depth understanding of the importance of meta-analyses to predict the risk associated with HCC. Traditional therapeutic strategies, including chemo and radiotherapy, are widely encouraged, but these strategies have limitations due to the resistance developed by tumor cells against chemotherapy drugs. Here, we focus on some important natural destructive mechanisms, like autophagy, and their roles. These mechanisms are dysregulated and promote tumorigenesis by enhancing chemotherapeutic resistance under aberrant conditions. Furthermore, the contributors have briefly compiled information and data regarding the current advanced clinical strategies such as immunotherapy and targeted therapy aimed specifically toward upregulated signalling pathways that are involved in cancer progression and angiogenesis. Finally, we explore current advances that personalized medicine aimed to benefit HCC patients. These novel medical strategies reflect current research concepts, and we are happy to present this issue to the scientific community with the hope that it will ultimately benefit cancer patients. 
Dr. G.P. Nagaraju is a faculty member in the Department of Hematology and Medical Oncology at Emory University School of Medicine. Dr. Nagaraju obtained his MSc and PhD, both in biotechnology from Sri Venkateswara University in Tirupati, Andhra Pradesh, India. Dr. Nagaraju received his DSc from Berhampur University in Berhampur, Odisha, India. Dr. Nagaraju's research focuses on translational research in gastrointestinal malignancies. He has published more than 95 research and review articles in highly respected international journals, and he has presented more than 50 abstracts at various national and international conferences. He is an author and editor of several books. Dr. Nagaraju is an editorial board member of several internationally recognized academic journals, and he is an associate member of the Discovery and Developmental Therapeutics research program at the Winship Cancer Institute. $\mathrm{He}$ is also a member of the Association of Scientists of Indian Origin in America (ASIOA), the Society for Integrative and Comparative Biology (SICB), the Science Advisory Board, The RNA
Society and the American Association of Cancer Research (AACR).

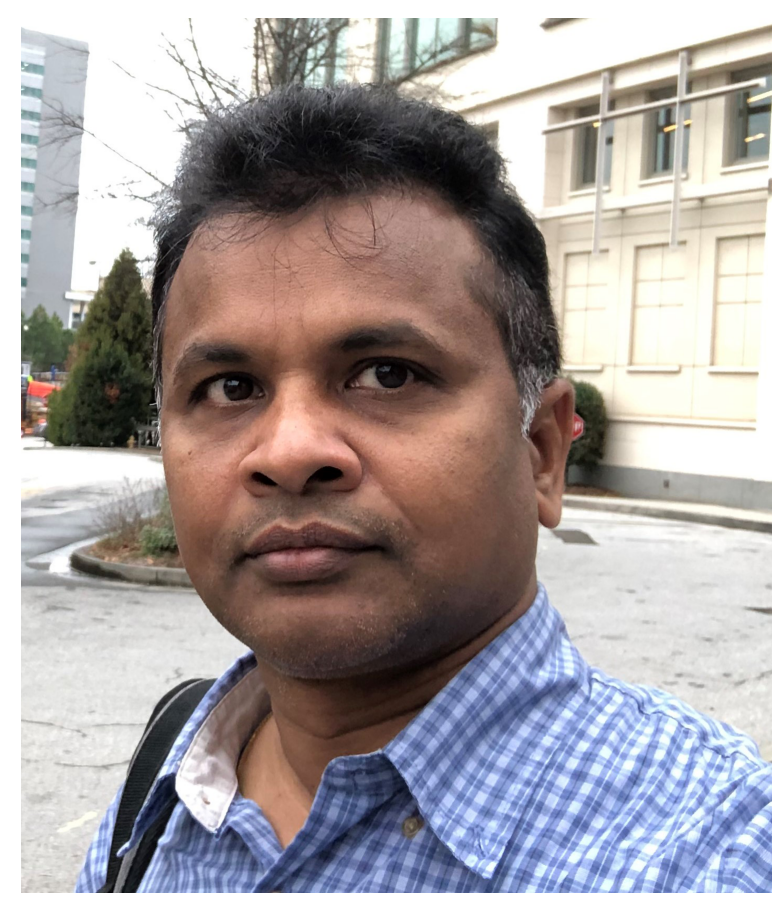

